\title{
Buona primavera ai Soci AIRP
}

Giornale di Tecniche Nefrologiche e Dialitiche 2019, Vol. 3I(2) III-III

(C) The Author(s) 2019

Article reuse guidelines:

sagepub.com/journals-permissions

DOI: 10.1 I77/0394936219847424

journals.sagepub.com/home/gtn

(S)SAE

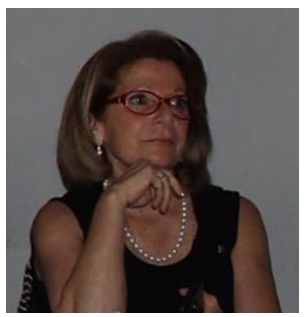

Cari Lettori e cari Amici,

un saluto affettuoso a tutti voi, come sempre, dalle pagine di GTND.

Desidero festeggiare con voi l'arrivo della primavera, la stagione che segna la fioritura del tulipano, il fiore che ci rappresenta! Abbiamo piantato i nostri bulbi all'inizio dell'inverno e ora stanno fiorendo spero a tutti.

Questi sono i miei, e ne vado molto fiera!

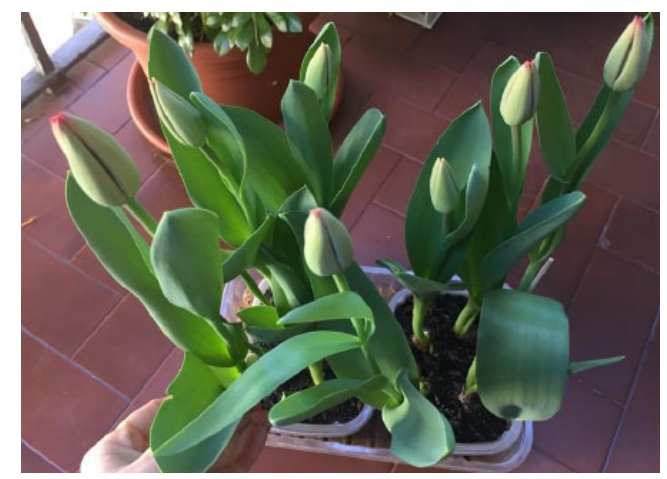

Figura I. "I miei tulipani rossi"

Quest'anno i nostri tulipani sono protagonisti di momenti di gioia come battesimi, cresime, comunioni, matrimoni e lauree e altre ricorrenze importanti. Possono essere un'occasione per compiere il vostro gesto di solidarietà in favore dei progetti AIRP.

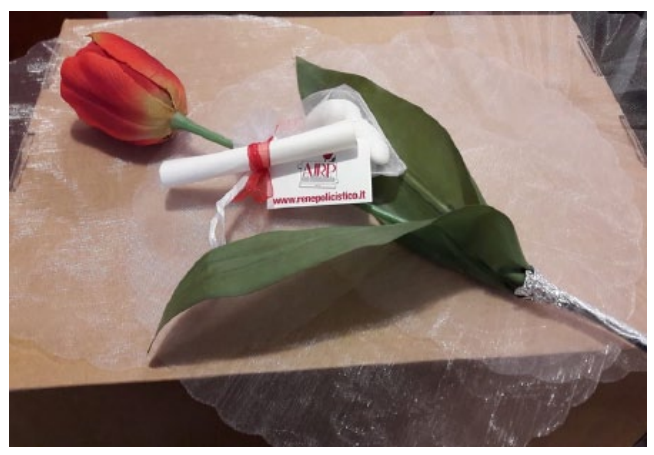

Non mi dilungo nel riassumere gli eventi del primo trimestre 2019 e quelli futuri perché li potrete trovare sul nostro sito www.renepolicistico.it.

Vi segnalo, come di consueto, gli articoli di questo numero: il primo di Flavia Galletti (Consigliere AIRP onlus e Responsabile Rapporti Internazionali) e di Franco Martini (Socio AIRP) che hanno partecipato il 16 marzo scorso al $1^{\circ}$ Summit Europeo dedicato ai pazienti affetti da $A D P K D$.

Il secondo articolo è di Emanuela Dorigo (Senior Project Manager T\&C srl) che relaziona sui risultati dell'indagine Il paziente con rene policistico negli ambulatori italiani.

Infine, per noi molto importante, desidero ricordarvi di rinnovare le quote di iscrizione all'Associazione e la donazione del $5 \times 1000$.

Ogni centesimo ricevuto da AIRP viene trasformato in impegno, attività, organizzazione, comunicazione, e ricerca. Per tutti noi.

Un caro saluto a tutti!

Luisa Sternfeld Pavia Presidente AIRP onlus

Presidente, AIRP onlus- Associazione Italiana Rene Policistico Onlus

\section{Corrispondenza:}

Dr.ssa Luisa Sternfeld Pavia, AIRP onlus,

Associazione Italiana Rene Policistico Onlus,

Via Bazzini 2, 20I3I Milano, Italy.

E-mail: luisa.sternfeld.airp@renepolicistico.it 